\title{
INVARIANT MEASURES AND CONTROLLABILITY OF FINITE SYSTEMS ON COMPACT MANIFOLDS
}

\author{
PHILIPPE JOUAN ${ }^{1}$
}

\begin{abstract}
A control system is said to be finite if the Lie algebra generated by its vector fields is finite dimensional. Sufficient conditions for such a system on a compact manifold to be controllable are stated in terms of its Lie algebra. The proofs make use of the equivalence theorem of [Ph. Jouan, ESAIM: COCV 16 (2010) 956-973]. and of the existence of an invariant measure on certain compact homogeneous spaces.
\end{abstract}

Mathematics Subject Classification. 17B66, 37A05, 37N35, 93B05, 93B17, 93C10.

Received February 11, 2011. Revised April 19, 2011.

Published online September 14, 2011.

It has been well known for a long time that a invariant control system on a compact Lie group is controllable if and only if it satisfies the rank condition (see [10]). This statement remains true for linear systems on compact Lie groups (see $[4,15])$.

On the other hand the equivalence theorem of [7] asserts that a control-affine system whose vector fields are complete and generate a finite dimensional Lie algebra is diffeomorphic to a linear system on a homogeneous space.

One might expect that under the rank condition a linear system on a compact homogeneous space is as well controllable. This statement is unfortunately not true, as shown by the counterexamples of Section 4 .

The purpose of this paper is to state sufficient controllability conditions for such systems, and thanks to the equivalence theorem, for systems whose Lie algebra $\mathcal{L}$ is finite dimensional. It makes use of the Poisson stability of invariant vector fields, and in certain cases of affine vector fields, on compact homogeneous spaces as soon as an invariant measure exists. The main result is as follows:

On a compact manifold $M$ consider a system that generates a finite dimensional Lie algebra $\mathcal{L}$, and satisfies the rank condition. It is controllable, hence finite time controllable, in the following cases:

- The algebra $\mathcal{L}$ is solvable.

- The algebra $\mathcal{L}$ is compact.

- Its zero-time ideal $\mathcal{L}_{0}$ is compact and its rank is maximum.

- The algebra $\mathcal{L}$ is semisimple and its dimension is equal to the one of $M$.

- Its zero-time ideal $\mathcal{L}_{0}$ is semisimple, its dimension and its rank are equal to the dimension of $M$.

It is moreover exact time controllable if and only if the rank of $\mathcal{L}_{0}$ is maximum.

\footnotetext{
Keywords and phrases. Compact homogeneous spaces, linear systems, controllability, finite dimensional Lie algebras, Haar measure.

${ }^{1}$ Lab. R. Salem, CNRS UMR 6085, Université de Rouen, avenue de l'Université, BP 12, 76801 Saint-Étienne-du-Rouvray, France. Philippe.Jouan@univ-rouen.fr
} 
These sufficient conditions of controllability hold for general, that is non control-affine, systems (Thm. $3.1 \mathrm{in}$ Sect. 3). However they are for technical reasons first proved for control-affine ones (Thm. 2.1 in Sect. 2).

In the two counterexamples provided in Section 4, the system algebra is semisimple non compact. Thus the semisimplicity of $\mathcal{L}$ is not sufficient for controllability, and additional conditions are required in that case.

Section 1 is devoted to some known definitions and facts from control theory.

\section{Preliminaries}

\subsection{Lie algebras and rank condition}

On a connected manifold $M$ consider the system

$$
\text { ( } \Xi) \quad \dot{p}=f(p, v)
$$

where $v$ belongs to a subset $V$ of $\mathbb{R}^{q}$, the mapping $f$ is continuous on $M \times V$ and $f_{v}=f(., v)$ is for every $v \in V$ a $\mathcal{C}^{k}$ vector field on $M$, with $k \geq 1$.

The set $\left\{f_{v} ; v \in V\right\}$ is denoted by $\Gamma$.

This family of vector fields is said to be transitive if the orbit through each point $p$ of $M$ is equal to $M$, that is if $M$ is the only orbit of $\Gamma$.

Let $V^{k}(M)$ stand for the space of $\mathcal{C}^{k}$ vector fields on $M$. It is not a Lie algebra whenever $k<+\infty$. But it may happen that all the Lie brackets of elements of $\Gamma$ of all finite lengths exist and are also $\mathcal{C}^{k}$. In that case the subspace of $V^{k}(M)$ spanned by these Lie brackets is a Lie algebra denoted by $\mathcal{L}(\Gamma)$ and we will say that the family $\Gamma$ generates a Lie algebra.

Let us assume that $\Gamma$ generates a Lie algebra, and let us consider the rank of $\Gamma$ at each point $p \in M$, that is the dimension of the subspace of $T_{p} M$, the tangent space to $M$ at $p$, spanned by the vectors $\gamma(p), \gamma \in \mathcal{L}(\Gamma)$. The so-called rank condition asserts that the family $\Gamma$ is transitive as soon as its rank is maximum, hence equal to $\operatorname{dim} M$, at each point.

More generally the family $\Gamma$ is said to be Lie-determined if at each point $p \in M$, the rank of $\Gamma$ at $p$ is equal to the dimension of the orbit of $\Gamma$ through $p$ (see for instance [9]).

We will denote by $\mathcal{L}_{0}$ the zero-time ideal of $\mathcal{L}$, that is the ideal generated by the differences $f_{u}-f_{v}, u, v \in \mathcal{U}$.

$\mathcal{L}$ and $\mathcal{L}_{0}$ are related by the equality

$$
\mathcal{L}=\mathbb{R} f(., u)+\mathcal{L}_{0}
$$

which holds for any $u \in V$.

\subsection{Controllability}

For $x \in M$ and $t \geq 0$, we denote by $\mathcal{A}(x, t)$ (resp. $\mathcal{A}(x, \leq t)$ ) the set of points of $M$ that can be reached from $x$ at time $t$ (resp. in time less than or equal to $t$ ). The system is said to be

$$
\text { controllable if } \quad \forall x \in N \quad \mathcal{A}(x)=\bigcup_{t \geq 0} \mathcal{A}(x, t)=M
$$

finite time controllable if $\exists T>0$ such that $\forall x \in N \quad \mathcal{A}(x, \leq T)=M$

exact time controllable if $\exists T>0$ such that $\forall x \in N \quad \mathcal{A}(x, T)=M$.

Let us recall the results of [8]:

The state space $M$ is assumed to be compact. If system $(\Xi)$ is controllable then it is finite time controllable.

In the case where the system is Lie determined, it is moreover exact time controllable if and only if the zero-time ideal has full rank at one point. 


\subsection{The Lie saturate}

Good references here are $[5,9]$.

A vector field $X$, the flow of which is denoted by $\left(\theta_{t}\right)_{t \in \mathbb{R}}$, belongs to the Lie saturate of $\Gamma$ if it belongs to $\mathcal{L}$ and verifies

where $\overline{\mathcal{A}(p)}$ is the closure of the reachable set from the point $p$.

$$
\forall p \in M \quad \forall t \geq 0 \quad \theta_{t}(p) \in \overline{\mathcal{A}(p)},
$$

The property that will be used further is the following:

The vector fields $\pm[X, Y]$ belong to the Lie saturate of the system as soon as this last contains $\pm X$ and $\pm Y$.

\subsection{The equivalence theorem}

For more details the reader is refered to [7].

A vector field on a connected Lie group $G$ is said to be linear if its flow is a one parameter group of automorphisms. To a linear vector field $\mathcal{X}$ one associates the derivation $D=-\operatorname{ad}(\mathcal{X})$ of the Lie algebra $\mathfrak{g}$ of $G$. In the case where this derivation is inner, that is $D=-\operatorname{ad}(X)$ for some right invariant vector field $X$ on $G$, the linear vector field splits into $\mathcal{X}=X+\mathcal{I}_{*} X$, where $\mathcal{I}$ stands for the diffeomorphism $g \in G \longmapsto \mathcal{I}(g)=g^{-1}$. Thus $\mathcal{X}$ is the sum of the right invariant vector field $X$ and the left invariant one $\mathcal{I}_{*} X$.

An affine vector field $F$ is obtained by adding a left invariant vector field $Z$ to a linear one $\mathcal{X}$. It may be more suitable to see $F$ as the sum of a linear vector field and a right invariant one. This can be done by writing:

$$
F=\mathcal{X}+Z=\mathcal{X}+Z+\mathcal{I}_{*} Z-\mathcal{I}_{*} Z=\tilde{\mathcal{X}}-\mathcal{I}_{*} Z
$$

where $\widetilde{\mathcal{X}}=\mathcal{X}+Z+\mathcal{I}_{*} Z$ is linear.

Let $H$ be a closed subgroup of $G$, and let $F=\mathcal{X}+Y$, where $Y$ is right invariant, be an affine vector field. The projection of $F$ onto the homogeneous space $G / H$ (manifold of left cosets of $H$ ) exists if and only if the subgroup $H$ is $\mathcal{X}$-invariant. In that case it will be refered to as an affine vector field on the homogeneous space $G / H$ (see [7] for the characterization of such vector fields).

A linear system on a Lie group or a homogeneous space is a system

$$
(L) \quad \dot{x}=F(x)+\sum_{j=1}^{m} u_{j} Y_{j}(x),
$$

where $F$ is an affine vector field and the $Y_{j}$ 's are right invariant if the state space is a Lie group, and projections of right invariant vector fields if the state space is a homogeneous space.

Let us consider the following system, defined on a connected (but not compact in general) manifold $M$ :

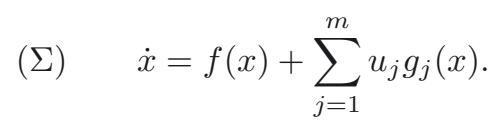

\section{Equivalence theorem [7].}

We assume the family $\left\{f, g_{1}, \ldots, g_{m}\right\}$ to be transitive. Then system $(\Sigma)$ is diffeomorphic to a linear system on a Lie group or a homogeneous space if and only if the vector fields $f, g_{1}, \ldots, g_{m}$ are complete and generate a finite dimensional Lie algebra.

More accurately, let $G$ (resp. $G_{0}$ ) be the connected and simply connected Lie group whose Lie algebra is $\mathcal{L}$ (resp. $\left.\mathcal{L}_{0}\right)$. Under the previous conditions the rank of $\mathcal{L}_{0}$ is constant, equal to $\operatorname{dim}(M)$ or $\operatorname{dim}(M)-1$, and:

(i) if rank $\left(\mathcal{L}_{0}\right)=\operatorname{dim}(M)$, in particular if there exists one point $p_{0} \in M$ such that $f\left(p_{0}\right)=0$, then $(\Sigma)$ is diffeomorphic to a linear system on a homogeneous space $G_{0} / H$ of $G_{0}$;

(ii) if rank $\left(\mathcal{L}_{0}\right)=\operatorname{dim}(M)-1$, then $\Sigma$ is diffeomorphic to an invariant system on a homogeneous space $G / H$ of $G$. 


\section{Controllability of CONTrol-Affine systems}

In this section the system under consideration is

$$
\text { ( }) \quad \dot{p}=f(p)+\sum_{j=1}^{m} u_{j} g_{j}(p),
$$

where

- $p$ belongs to a compact and connected manifold $M$;

- the vector fields $f$ and $g_{1}, \ldots, g_{m}$ are $\mathcal{C}^{k}, k \geq 1$;

- the controls $u_{1}, \ldots, u_{m}$ take their values in $\mathbb{R}^{m}$;

- the admissible inputs are the piecewise constant ones. More generally the forthcoming Theorem 2.1 remains true when the set of admissible inputs is a subspace of $L^{\infty}\left(\mathbb{R}_{+}, \mathbb{R}^{m}\right)$ which contains the piecewise constant ones.

The manifold $M$ being compact, the vector fields $f, g_{1}, \ldots, g_{m}$ are complete.

In order to apply the equivalence theorem they are moreover assumed to generate a finite dimensional Lie algebra $\mathcal{L}$ and to satisfy the rank condition. According to this theorem, and with its notations, system $(\Sigma)$ is diffeomorphic to an invariant system on a homogeneous space $G / H$ of $G$ if $\operatorname{rank}\left(\mathcal{L}_{0}\right)=\operatorname{dim}(M)-1$ or $\operatorname{rank}\left(\mathcal{L}_{0}\right)=\operatorname{dim}(M)$ but $\mathcal{L}_{0}=\mathcal{L}$, and diffeomorphic to a linear system on a homogeneous space $G_{0} / H$ of $G_{0}$ if $\operatorname{rank}\left(\mathcal{L}_{0}\right)=\operatorname{dim}(M)$ and $\mathcal{L}_{0} \neq \mathcal{L}$.

In the second case $M$ is as well diffeomorphic to a homogeneous space $G / H$ of $G$, and $(\Sigma)$ equivalent to an invariant system on $G / H$. This remark will be used when $\mathcal{L}$ and $\mathcal{L}_{0}$ will be solvable.

However the nature of the Lie algebras $\mathcal{L}$ and $\mathcal{L}_{0}$ of $G$ and $G_{0}$ are different in general, and it may be more worthwhile to consider $\mathcal{L}_{0}$ rather than $\mathcal{L}$. It will be the case when $\mathcal{L}_{0}$ will be semisimple or compact.

Notice that the system lifted on $G$ from $(\Sigma)$ cannot be controllable whenever $\mathcal{L}_{0} \neq \mathcal{L}$. Indeed $G$ is simply connected, and all the system vector fields are contained in a half space bounded by the Lie algebra $\mathcal{L}_{0}$ (hypersurface principle, see [14]).

System $(\Sigma)$ is of course controllable as soon as the system lifted on $G$ (or on $G_{0}$ ) is controllable (this includes the cases $f=0$ and $M$ diffeomorphic to a compact Lie group).

In this paper the controllability of $(\Sigma)$ will be proved by showing the Poisson stability of the vector field $f$. The main result is the following:

Theorem 2.1. The vector fields $f, g_{1}, \ldots, g_{m}$ are assumed to generate a finite dimensional Lie algebra $\mathcal{L}$, and to satisfy the rank condition at every point of $M$.

Then system $(\Sigma)$ is controllable, hence finite time controllable, in the following cases:

(1) The algebra $\mathcal{L}$ is solvable.

(2) The algebra $\mathcal{L}$ is compact.

(3) The algebra $\mathcal{L}_{0}$ is compact and its rank is maximum.

(4) The algebra $\mathcal{L}$ is semisimple and its dimension is equal to the one of $M$.

(5) The algebra $\mathcal{L}_{0}$ is semisimple, its dimension and its rank are equal to the dimension of $M$.

It is moreover exact time controllable if and only if the rank of $\mathcal{L}_{0}$ is maximum.

Proof. Controllability under one of the conditions 1-5 is proved in Sections 2.2-2.4. Let us investigate the finite time and exact time controllability properties. According to the results of [8], recalled in Section 1.2, system $(\Sigma)$ is finite time controllable as soon as it is controllable, because of the compactness of the state space. On account of the equivalence theorem the rank of the zero-time ideal $\mathcal{L}_{0}$ is constant and the system is Lie determined. It is therefore exact time controllable if and only if the rank of $\mathcal{L}_{0}$ is equal to the dimension of $M$. 


\subsection{Invariant measures and Poisson stability}

The idea to consider stability according to Poisson and one of its possible causes, the invariance of a finite measure, in order to prove controllability goes back to [11].

Let $f$ be a complete vector field, the flow of which is denoted by $\left(\varphi_{t}\right)_{t \in \mathbb{R}}$, on a connected manifold $M$. The point $p \in M$ is said to be positively Poisson stable (resp. negatively Poisson stable) (resp. Poisson stable) if for every neighbourhood $V$ of $p$ and all $T>0$ there exists $t \geq T$ such that $\varphi_{t}(p) \in V\left(\operatorname{resp} . \varphi_{-t}(p) \in V\right)(\operatorname{resp}$. if it is positively and negatively stable).

The vector field $f$ is said to be Poisson stable if the set of Poisson stable points is dense in $M$.

A measure $\mu$ on $M$ is said to be invariant under the action of the flow of $f$, or equivalently the vector field $f$ is said to be measure preserving, if for every measurable set $A$ and all $t \in \mathbb{R}, \mu\left(\varphi_{t}(A)\right)=\mu(A)$.

Theorem A. Let $f$ be a complete vector field on a connected manifold $M$. The field $f$ is assumed to preserve a finite Borel measure. Then the measure of the set of non Poisson stable points of $M$ is zero.

Proof. See [13] for instance.

Whenever the measure of the nonempty open sets does not vanish, the conclusion of Theorem A implies the Poisson stability of $f$.

Theorem B. We assume that $(\Sigma)$ satisfies the rank condition, and that the vector field $f$ is Poisson stable. Then the vector field $-f$ belongs to the Lie saturate of $(\Sigma)$.

Proof. See [5] for instance.

Let us assume the hypothesis of Theorem B to be satisfied. Then the vector fields $\pm f$ and $\pm g_{j}$ for $j=1, \ldots, m$ belong to the Lie saturate of the system. But according to a theorem recalled in Section 1.3, the vector fields $\pm[X, Y]$ belong to the Lie saturate of the system as soon as this last contains $\pm X$ and $\pm Y$. By an obvious induction the Lie saturate of $(\Sigma)$ is equal to $\mathcal{L}$ and for all $p \in M$ the closure of $\mathcal{A}(p)$ is equal to $M$. According to Corollary 2, p. 69 of [9], and under the rank condition, this implies $\mathcal{A}(p)=M$. The system is therefore controllable under the assumptions of Theorem B.

It is henceforth of interest to answer the two following questions. Let $G$ be a connected Lie group and let $H$ be a closed subgroup of $G$ such that the quotient $G / H$ is compact. A $G$-invariant, or invariant in short, measure on $G / H$ is a positive Radon measure preserved by the left action of $G$ on $G / H$.

(1) Does the Haar measure of $G$ induce an invariant, finite measure on $G / H$ ? If such a measure exists, it is preserved by the invariant vector fields of $G / H$. The measure of the non empty open sets being positive the invariant fields are Poisson stable.

(2) If the answer to the first question is positive, is the $G$-invariant measure of $G / H$ preserved by the affine vector fields of $G / H$ ?

The answer to the first question is related to the modular function of the group $G$. Let us recall its definition: let $\mu_{l}$ and $\mu_{r}$ be respectively the left and right Haar measures on $G$. They are defined up to a positive constant and verify

$$
\mu_{r}(\mathrm{~d} g)=\Delta_{G}(g) \mu_{l}(\mathrm{~d} g)
$$

where $\Delta_{G}$ is a continuous morphism from $G$ into the multiplicative group $\mathbb{R}_{+}^{*}=\{\alpha>0\}$, called modular function. This definition extends to locally compact topological groups, and whenever $G$ is a Lie group, we have

$$
\forall g \in G \quad \Delta_{G}(g)=|\operatorname{det}(\operatorname{Ad}(g))|,
$$

where $\operatorname{Ad}(g)$ stands for the differential at the identity $e$ of the mapping $x \longmapsto g x g^{-1}$. The following result can be found for instance in [6] or [1]. The (left) Haar measures of $G$ and $H$ are respectively denoted by $\mu_{G}$ and $\mu_{H}$, and their modular functions by $\Delta_{G}$ and $\Delta_{H}$. 
Theorem C. Let $H$ be a closed subgroup of the Lie group $G$. There exists a $G$-invariant measure on $G / H$ if and only if

$$
\forall h \in H \quad \Delta_{G}(h)=\Delta_{H}(h) .
$$

This measure, unique up to a positive constant and denoted by $\mu_{G / H}$, is defined by the following equality: for every compactly supported continuous function $\theta$ on $G$,

$$
\int_{G} \theta(g) \mu_{G}(\mathrm{~d} g)=\int_{G / H}\left(\int_{H} \theta(\mathrm{g} h) \mu_{H}(\mathrm{~d} h)\right) \mu_{G / H}(\mathrm{~d}(g H)) .
$$

The second statement of Theorem $\mathrm{C}$ is related to the following lemma, proved in [6] and which will be used in Section 2.3.

Lemma A. Let $\mathcal{C}_{c}(G)$ (resp. $\mathcal{C}_{c}(G / H)$ ) denote the set of compactly supported continuous functions of $G$ (resp. $G / H)$. The mapping $\theta \longmapsto P(\theta)$ from $\mathcal{C}_{c}(G)$ to $\mathcal{C}_{c}(G / H)$ defined by

$$
P(\theta)(g H)=\int_{H} \theta(g h) \mu_{H}(\mathrm{~d} h)
$$

is onto.

The group $G$ is said to be unimodular if its modular function is constant, equal to 1 . Theorem C applies in particular, but not only, when the groups $G$ and $H$ are unimodular. In the previously quoted references it is shown that are unimodular:

- the groups whose adjoint group is compact, in particular the compact and discrete groups;

- the semisimple groups;

- the connected nilpotent groups.

\subsection{Proof of the solvable case}

Let us first remark that the Lie algebra $\mathcal{L}$ is solvable if and only if its subalgebra $\mathcal{L}_{0}$ is. Indeed a subalgebra of a solvable Lie algebra is solvable. Conversely the equality $\mathcal{L}=\mathbb{R} f+\mathcal{L}_{0}$ and the stability of $\mathcal{L}_{0} \operatorname{under}$ ad $(f)$ imply that the derived algebra of $\mathcal{L}$ is contained in $\mathcal{L}_{0}$, and therefore that $\mathcal{L}$ is solvable whenever $\mathcal{L}_{0}$ is.

The algebras $\mathcal{L}$ and $\mathcal{L}_{0}$ being assumed to be solvable the manifold $M$ is diffeomorphic to an homogeneous space $G / H$ of $G$, and $f$ is equivalent to an invariant vector field. According to a theorem of Mostow [12] a compact solv manifold, that is a compact homogeneous space of a solvable Lie group, has a $G$-invariant measure. This measure is invariant under the action of $G$ on $G / H$, hence preserved by the flow of $f$. According to Section 2.1 the field $f$ is Poisson stable, and the system controllable.

\subsection{Proof of the semisimple case}

Whenever the system algebra is semisimple one cannot expect to obtain results as general as in the solvable case. The main obstruction is that the quotient $G / H$ of a semisimple Lie group does not always have an invariant measure, even if it is compact. In Section 4 two examples of invariant systems on a compact homogeneous space of a semisimple Lie group that are not controllable, although they satisfy the rank condition, are exhibited.

However semisimple and discrete groups being unimodular, the homogeneous space of a semisimple group by a discrete subgroup $H$ has an invariant measure. The discrete subgroup $H$ is said to be a uniform lattice if the quotient $G / H$ is compact. Borel showed in [3] that every semisimple connected group possesses a uniform lattice. This notion is therefore relevant for our purpose.

The algebras $\mathcal{L}$ and $\mathcal{L}_{0}$ cannot be both semisimple without being equal: otherwise $\mathcal{L}_{0}$ is a codimension one ideal of $\mathcal{L}$, which is impossible whenever $\mathcal{L}$ is semisimple. Therefore we have to distinguish two possibilities. 
(1) $\mathcal{L}$ is semisimple and its dimension is equal to the one of $M$. In that case $M$ is diffeomorphic to a quotient of $G$ by a uniform lattice $H$, and $(\Sigma)$ to an invariant system on $G / H$. As $G / H$ has an invariant measure, this invariant system is controllable, and even exact time controllable since $\mathcal{L}_{0}=\mathcal{L}$.

(2) $\mathcal{L}_{0}(\neq \mathcal{L})$ is semisimple, its dimension and its rank are equal to the dimension of $M$. In that case $M$ is diffeomorphic to the quotient of $G_{0}$ by a uniform lattice $H$, and $(\Sigma)$ is equivalent to a linear system on $G_{0} / H$. As $\mathcal{L} \neq \mathcal{L}_{0}$ the system cannot be reduced to an invariant one, and we have to prove that the invariant measure of $G_{0} / H$ is preserved by the affine vector fields.

Proposition 2.1. Let $F$ be an affine vector field on a semisimple connected Lie group $G_{0}$, and $F=\mathcal{X}+Y$ its decomposition into a linear vector field $\mathcal{X}$ and a right invariant one $Y$. The projection of $\mathcal{X}$ onto $G_{0} / H$, where $H$ is a uniform lattice of $G_{0}$, is assumed to exist.

Then the projection of $F$ onto $G_{0} / H$ preserves the invariant measure of $G_{0} / H$.

Proof. As $G_{0}$ is semisimple there exists a right invariant vector field $X$ such that $\mathcal{X}=X+\mathcal{I}_{*} X$. The field $F$ is therefore equal to $W+\mathcal{I}_{*} X$, where $W=X+Y$ is right invariant and $\mathcal{I}_{*} X$ left invariant. Its flow, denoted by $\left(\eta_{t}\right)_{t \in \mathbb{R}}$, is thus

$$
(g, t) \longmapsto \exp (t W) g \exp (-t X) .
$$

The flow of its projection onto $G_{0} / H$ is denoted by $\left(\bar{\eta}_{t}\right)_{t \in \mathbb{R}}$, and with obvious notations these two flows are related by

$$
\forall g \in G_{0} \quad \forall t \in \mathbb{R} \quad \bar{\eta}_{t}(g H)=\eta_{t}(g) H .
$$

Let $a$ be a compactly supported continuous function on $G_{0}$ and, with the notations of Lemma A, let us first show that

$$
P\left(a \circ \eta_{t}\right)=P(a) \circ \bar{\eta}_{t}
$$

The subgroup $H$ being discrete it is included in the set of fixed points of $\mathcal{X}$, that is $\forall t \in \mathbb{R}$ and $\forall h \in H$, $\exp (t X) h \exp (-t X)=h$ (see Sect. 1.4). This allows to state, with $A=P(a) \in \mathcal{C}_{c}(G / H)$ :

$$
\begin{aligned}
\int_{H} a \circ \eta_{t}(g h) \mu_{H}(\mathrm{~d} h) & =\int_{H} a(\exp (t W) g h \exp (-t X)) \mu_{H}(\mathrm{~d} h) \\
& =\int_{H} a(\exp (t W) g \exp (-t X) h) \mu_{H}(\mathrm{~d} h) \\
& =\int_{H} a\left(\eta_{t}(g) h\right) \mu_{H}(\mathrm{~d} h) \\
& =A\left(\eta_{t}(g) H\right)=A \circ \bar{\eta}_{t}(g H) .
\end{aligned}
$$

The measure $\mu_{G_{0}}$ being unimodular, hence left and right invariant, we obtain

$$
\begin{aligned}
\int_{G_{0} / H} A \circ \bar{\eta}_{t}(g H) \mu_{G_{0} / H}(\mathrm{~d}(g H)) & =\int_{G_{0}} a \circ \eta_{t}(g) \mu_{G_{0}}(\mathrm{~d} g) \\
& =\int_{G_{0}} a(\exp (t W) g \exp (-t X)) \mu_{G_{0}}(\mathrm{~d} g) \\
& =\int_{G_{0}} a(g) \mu_{G_{0}}(\mathrm{~d} g) \\
& =\int_{G_{0} / H} A(g H) \mu_{G_{0} / H}(\mathrm{~d}(g H)) .
\end{aligned}
$$

This shows that the measure $\mu_{G_{0} / H}$ is preserved by the projection of $F$. 


\subsection{Proof of the compact case}

Let $G$ be a connected and simply connected Lie group whose Lie algebra $\mathfrak{g}$ is compact. The adjoint group $\operatorname{Ad}_{G}(G)$ being compact the group $G$ is unimodular.

Let us show that every subgroup $H$ of $G$, closed but not necessarily connected, is also unimodular. Let $W$ be a compact neighbourhood of the identity in $G$ such that $\operatorname{Ad}_{G}(W)=\operatorname{Ad}_{G}(G)$ (the existence of $W$ is proved in [1], p. 108). The intersection $H \cap W$ being compact, $\operatorname{Ad}_{G}(H)=\operatorname{Ad}_{G}(H \cap W)$ is a compact subgroup of $G l(\mathfrak{g})$. It remains to prove that the group $\operatorname{Ad}_{H}(H)$, that is the set of restrictions to the Lie algebra $\mathfrak{h}$ of $H$ of the elements of $\operatorname{Ad}_{G}(H)$, is also compact. Let us consider a direct sum $\mathfrak{g}=\mathfrak{h} \oplus v$, let us denote by $p$ the projection of $\mathfrak{g}$ onto $\mathfrak{h}$ along $v$, and by $i$ the canonical injection of $\mathfrak{h}$ into $\mathfrak{g}$. Then the mapping $\Theta$ defined by

$$
\Theta(\rho)=p \circ \rho \circ i
$$

is continuous from $\operatorname{Ad}_{G}(H)$ onto $\operatorname{Ad}_{H}(H)$, which is thus compact.

This shows that the group $H$ is unimodular, and that the quotient $G / H$ has an invariant measure, finite if the quotient is compact.

As previously, two cases are considered.

(1) The algebra $\mathcal{L}$ is compact. In that case $M$ is diffeomorphic to the quotient of $G$ by a closed subgroup $H$, and $(\Sigma)$ is equivalent to an invariant system on $G / H$. Since $G / H$ possesses an invariant measure, this last is controllable.

(2) The algebra $\mathcal{L}_{0}$ is compact, and its rank is equal to the dimension of $M$. In that case $M$ is diffeomorphic to the quotient of $G_{0}$ by a closed subgroup $H,(\Sigma)$ is equivalent to a linear system on $G_{0} / H$, and we have to show that the invariant measure of $G_{0} / H$ is preserved by the affine vector fields.

Proposition 2.2. Let $H$ be a closed subgroup of the connected Lie group $G$ whose Lie algebra is compact. Let $N$ be the normalizer of $H$ in $G$, and for $x \in N$ let $\rho_{x}$ be the diffeomorphism of $G / H$ defined by $\rho_{x}(g H)=g x H$. The invariant measure of $G / H$ is preserved by $\rho_{x}$ for all $x \in N$.

Proof. We denote by $\mathcal{C}_{c}(G / H)$ the set of compactly supported continuous functions of $G / H$. For $A \in \mathcal{C}_{c}(G / H)$ we set

$$
I(A)=\int_{G / H} A(g H) \mu_{G / H}(\mathrm{~d}(g H)) \quad \text { and } \quad J_{x}(A)=I\left(A \circ \rho_{x}\right) .
$$

For $y \in G$ the diffeomorphism $\lambda_{y}$ of $G / H$ is defined by $\lambda_{y}(g H)=y g H$. It is clear that $\lambda_{y} \circ \rho_{x}=\rho_{x} \circ \lambda_{y}$ for all $x \in N$ and all $y \in G$. It follows that $\forall A \in \mathcal{C}_{c}(G / H), \forall y \in G$

$$
J_{x}\left(A \circ \lambda_{y}\right)=I\left(A \circ \lambda_{y} \circ \rho_{x}\right)=I\left(A \circ \rho_{x} \circ \lambda_{y}\right)=I\left(A \circ \rho_{x}\right)=J_{x}(A) .
$$

This shows that $J_{x}$ is a $G$-invariant, positive, Radon measure, hence equal to $\delta(x) I$ for some positive constant $\delta(x)$.

We have to show that this constant is equal to 1 for all $x \in N$.

The measure $\mu_{G / H}$ is defined by a density of $G / H$ also denoted by $\mu_{G / H}$ (cf. Appendix). Then, denoting $\Gamma_{x}=\lambda_{x} \circ \rho_{x^{-1}}$, we have

$$
J_{x}(A)=I\left(A \circ \Gamma_{x^{-1}}\right)=\int_{G / H} A \circ \Gamma_{x^{-1}} \mu_{G / H}=\int_{G / H} A\left(\Gamma_{x}\right)^{*} \mu_{G / H},
$$

hence $\delta(x) \mu_{G / H}=\left(\Gamma_{x}\right)^{*} \mu_{G / H}$. As $\Gamma_{x}(H)=H$ we obtain $\delta(x)=\left|\operatorname{det}\left(T_{H} \Gamma_{x}\right)\right|$.

Let $\gamma_{x}$ stand for the diffeomorphism $g \longmapsto x g x^{-1}$, and $\pi$ for the projection of $G$ onto $G / H$. For $x \in N$ we have $\pi \circ \gamma_{x}=\Gamma_{x} \circ \pi$ hence

$$
\operatorname{det}\left(T_{H} \Gamma_{x}\right)=\frac{\operatorname{det}\left(\operatorname{Ad}_{G}(x)\right)}{\operatorname{det}\left(\left.\operatorname{Ad}_{G}(x)\right|_{H}\right)}=\frac{1}{\operatorname{det}\left(\left.\operatorname{Ad}_{G}(x)\right|_{H}\right)},
$$


the last equality being due to the fact that $G$ is unimodular. Now $x \in N \longmapsto \operatorname{det}\left(\left.\operatorname{Ad}_{G}(x)\right|_{H}\right)$ is a continuous morphism from $N$ into $\mathbb{R}^{*}$. Moreover the subgroup $N$ is closed in $G$ because it is the normalizer of the closed subgroup $H$. The group $\operatorname{Ad}_{G}(N)$ is therefore compact and $\operatorname{det}\left(\left.\operatorname{Ad}_{G}(N)\right|_{H}\right)$ is contained in $\{-1,1\}$.

Corollary 2.1. Let $G_{0} / H$ be the compact quotient of a connected Lie group $G_{0}$ whose Lie algebra is compact, by a closed subgroup $H$. Then the invariant measure of $G_{0} / H$ is preserved by every affine vector field $f$ of $G_{0} / H$.

Proof. Let $F$ be an affine vector field of $G_{0}$, the projection of which onto $G_{0} / H$ is equal to $f$.

The Lie algebra $\mathfrak{g}$ of $G_{0}$ is equal to $z(\mathfrak{g}) \oplus[\mathfrak{g}, \mathfrak{g}]$, where $z(\mathfrak{g})$ is the center of $\mathfrak{g}$ and $[\mathfrak{g}, \mathfrak{g}]$ semisimple compact, and the derivation associated to $F$ splits into $A \oplus \operatorname{ad}\left(X_{1}\right)$ where $A$ is an endomorphism of $z(\mathfrak{g})$ and $X_{1} \in[\mathfrak{g}, \mathfrak{g}]$. In the same way $G_{0}$ is equal to the direct product $\mathbb{R}^{n} \times G_{1}$ where $G_{1}$ is a semisimple compact Lie group. The quotient $G_{0} / H$ being compact, the subgroup $\mathbb{R}^{n} \cap H$ is up to an isomorphism equal to $\mathbb{Z}^{n}$. But this set is $\mathrm{e}^{t A}$ invariant for $t \in \mathbb{R}$, and $A$ vanishes.

The derivation associated to $F$ is therefore inner, and $F$ splits into $F=\mathcal{X}+Y$ where $Y$ is a right invariant vector field and $\mathcal{X}=X+\mathcal{I}_{*} X$, with $X$ right invariant, is linear. As well as in the semisimple case the vector field $F$ is eventually equal to $W+\mathcal{I}_{*} X$ where $W=X+Y$ is right invariant and $\mathcal{I}_{*} X$ is left invariant. The flow of its projection onto $G / H$ is equal to

$$
(g H, t) \longmapsto \exp (t W) g \exp (-t X) H
$$

Because of the existence of this projection, the subgroup $H$ is invariant under the flow $(g, t) \longmapsto \exp (t X) g$ $\exp (-t X)$ of $\mathcal{X}$ (see Sect. 1.4). Consequently $\exp (t X)$ belongs to the normalizer of $H$ for all $t \in \mathbb{R}$, and according to Proposition 2.2, $f$ preserves the measure $\mu_{G / H}$.

\section{Extension to NON CONTROL-AFFine SySTEMS}

On the compact and connected manifold $M$ let us now consider the system

$$
(\Xi) \quad \dot{p}=f(p, v)
$$

where $v$ belongs to a subset $V$ of $\mathbb{R}^{q}$, the mapping $f$ is continuous on $M \times V$ and $f_{v}=f(., v)$ is for every $v \in V$ a $\mathcal{C}^{k}$ vector field on $M$, with $k \geq 1$.

The set $\Gamma=\left\{f_{v} ; v \in V\right\}$ of vector fields of the system is assumed to generate a finite dimensional Lie algebra $\mathcal{L}$ and to satisfy the rank condition.

We denote by $\Gamma_{0}=\left\{f_{v_{1}}-f_{v_{2}} ; v_{1}, v_{2} \in V\right\}$ the set of differences of elements of $\Gamma$ and as usual by $\mathcal{L}_{0}$ the ideal of $\mathcal{L}$ generated by $\Gamma_{0}$.

Let us choose $f_{0} \in \Gamma$, arbitrarily if the vector spaces $\operatorname{Vect}(\Gamma)$ and $\operatorname{Vect}\left(\Gamma_{0}\right)$ respectively generated by $\Gamma$ and $\Gamma_{0}$ are distinct, and otherwise equal to 0 . Then let us also choose vector fields $f_{v_{1}}, \ldots, f_{v_{m}}$ in $\Gamma$ such that the fields $g_{1}, \ldots, g_{m}$, where $g_{i}=f_{v_{i}}-f_{0}$, be a basis of $\operatorname{Vect}\left(\Gamma_{0}\right)$. For all $v \in V$ there exist real numbers $u_{1}, \ldots, u_{m}$ such that $f_{v}$ writes

$$
f_{v}=f_{0}+\sum_{j=1}^{m} u_{j} g_{j}
$$

Consider the control affine system

$$
\dot{p}=f_{0}(p)+\sum_{j=1}^{m} u_{j} g_{j}(p) \quad\left(u_{1}, \ldots, u_{m}\right) \in \mathbb{R}^{m} .
$$

Its Lie algebra is equal to $\mathcal{L}$, the ideal generated by $g_{1}, \ldots, g_{m}$ is equal to $\mathcal{L}_{0}$, and $(\Sigma)$ satisfies the rank condition. 
If $\mathcal{L}$ or $\mathcal{L}_{0}$ satisfies one of the conditions of Theorem 2.1 then all the vector fields of $(\Sigma)$, hence all the vector fields of $(\Xi)$, are Poisson stable. Thus the Lie saturate of $(\Xi)$ contains $\pm f_{v}$ for all $v \in V$. It is therefore equal to $\mathcal{L}$ and $(\Xi)$ is controllable. We have proved:

Theorem 3.1. The vector fields $f_{v}, v \in V$, are assumed to generate a finite dimensional Lie algebra $\mathcal{L}$, and to satisfy the rank condition at every point of $M$.

Then system $(\Xi)$ is controllable, hence finite time controllable, in the following cases:

(1) The algebra $\mathcal{L}$ is solvable.

(2) The algebra $\mathcal{L}$ is compact.

(3) The algebra $\mathcal{L}_{0}$ is compact and its rank is maximum.

(4) The algebra $\mathcal{L}$ is semisimple and its dimension is equal to the one of $M$.

(5) The algebra $\mathcal{L}_{0}$ is semisimple, its dimension and its rank are equal to the dimension of $M$.

It is moreover exact time controllable if and only if the rank of $\mathcal{L}_{0}$ is maximum.

Important remark. Theorem 2.1 was stated for unbounded, piecewise constant inputs. On account of Theorem 3.1 it remains true if the set of admissible inputs is restricted to bounded, or bang-bang, piecewise constant ones. Indeed we can consider such a system as a non control-affine one and apply Theorem 3.1.

\section{Counterexamples}

\subsection{Counterexample on the sphere $S^{1}$}

The one dimensional sphere $S^{1}$ is identified with $\mathbb{R} / 2 \pi \mathbb{Z}$. The vector fields $C, S$ and $T$ are the projections onto $S^{1}$ of the vector fields $\cos t \frac{\mathrm{d}}{\mathrm{d} t}, \sin t \frac{\mathrm{d}}{\mathrm{d} t}$ and $\frac{\mathrm{d}}{\mathrm{d} t}$ of the real line. The Lie brackets of these vector fields are:

$$
[C, S]=T, \quad[C, T]=S, \quad[T, S]=C,
$$

hence $(C, T, S)$ is the basis of a 3 -dimensional Lie algebra $\mathfrak{g}$.

We consider on $S^{1}$ the system

$$
\dot{p}=C(p)+u S(p),
$$

where $u \in \mathbb{R}$. The field $S$ vanishes at 0 and $\pi$, and at these two points the field $C$ points toward the interior of the interval ] $0, \pi\left[\right.$ ( or to the same halfspace in $\mathbb{R}^{2}$ ). This shows that $(\Sigma)$ cannot be controllable though its Lie algebra $\mathcal{L}$ is equal to $\mathfrak{g}$ (as well as the ideal $\mathcal{L}_{0}$ ) and satisfies the rank condition.

We can notice that $\mathfrak{g}$ is isomorphic to $\mathfrak{s l}_{2}$. Indeed that Lie algebra is generated by the matrices

$$
H=\left(\begin{array}{cc}
1 & 0 \\
0 & -1
\end{array}\right), \quad E=\left(\begin{array}{ll}
0 & 1 \\
0 & 0
\end{array}\right), \quad F=\left(\begin{array}{ll}
0 & 0 \\
1 & 0
\end{array}\right)
$$

which satisfy

The isomorphism is defined by

$$
[H, E]=2 E, \quad[H, F]=-2 F, \quad[E, F]=H .
$$

$$
2 C \sim H \quad S+T \sim E \quad S-T \sim F .
$$

At the point $p=\frac{\pi}{2}$ the field $C$ vanishes and the fields $S$ and $T$ take the same value. The sphere $S^{1}$ can therefore be viewed as the quotient of $S L_{2}$ by a subgroup $K$ whose Lie algebra is generated by $H$ et $F$. The connected group generated by these matrices is

$$
\widetilde{K}=\left\{\left(\begin{array}{cc}
a & 0 \\
b & a^{-1}
\end{array}\right) ; \quad(a, b) \in \mathbb{R}_{+}^{*} \times \mathbb{R}\right\} .
$$

It is not unimodular (this can be verified directly). Otherwise the quotient of $S L_{2}$ by $\widetilde{K}$ would have a $S L_{2}$ invariant measure, such a measure would also exist on $S^{1}$ and the system would be controllable.

Finally notice that the algebra $\mathcal{L}$ is semisimple non compact, and that the field $C$ is not Poisson stable. 


\subsection{Counterexample on the sphere $S^{2}$}

If the sphere $S^{1}$ is embedded into $\mathbb{R}^{2}$ :

$$
S^{1}=\left\{(x, y) \in \mathbb{R}^{2} ; x^{2}+y^{2}=1\right\}
$$

then the field $C$ (resp. $S$ ) is at the point $(x, y) \in S^{1}$ the orthogonal projection to the tangent space of $S^{1}$ at $(x, y)$ of the constant field $\frac{\partial}{\partial y}$ (resp. $-\frac{\partial}{\partial x}$ ) of $\mathbb{R}^{2}$. The field $T$ is equal to $-y \frac{\partial}{\partial x}+x \frac{\partial}{\partial y}$.

We can build in the same way an example on the sphere $S^{2}$. Let $T_{x}, T_{y}$ and $T_{z}$ be the orthogonal projections onto $S^{2}$ of the constant fields $\frac{\partial}{\partial x}, \frac{\partial}{\partial y}$ and $\frac{\partial}{\partial z}$ of $\mathbb{R}^{3}$, and $R_{x}, R_{y}, R_{z}$ be the restrictions to $S^{2}$ of the rotations about the axis $O x, O y$ and $O z$.

In the natural coodinates of $\mathbb{R}^{3}$ these fields write

$$
\begin{array}{ccc}
T_{x}=\left(\begin{array}{c}
1-x^{2} \\
-x y \\
-x z
\end{array}\right), & T_{y}=\left(\begin{array}{c}
-y x \\
1-y^{2} \\
-y z
\end{array}\right), & T_{z}=\left(\begin{array}{c}
-z x \\
-z y \\
1-z^{2}
\end{array}\right), \\
R_{x}=\left(\begin{array}{c}
0 \\
-z \\
y
\end{array}\right), & R_{y}=\left(\begin{array}{c}
z \\
0 \\
-x
\end{array}\right), & R_{z}=\left(\begin{array}{c}
-y \\
x \\
0
\end{array}\right) .
\end{array}
$$

Their Lie brackets are

$$
\begin{aligned}
{\left[T_{x}, T_{y}\right] } & =\left[R_{y}, R_{x}\right]=R_{z}, \\
{\left[T_{y}, T_{z}\right] } & =\left[R_{z}, R_{y}\right]=R_{x}, \\
{\left[T_{z}, T_{x}\right] } & =\left[R_{x}, R_{z}\right]=R_{y}, \\
{\left[T_{x}, R_{y}\right] } & =\left[R_{x}, T_{y}\right]=-T_{z}, \\
{\left[T_{y}, R_{z}\right] } & =\left[R_{y}, T_{z}\right]=-T_{x}, \\
{\left[T_{z}, R_{x}\right] } & =\left[R_{z}, T_{x}\right]=-T_{y}, \\
{\left[T_{x}, R_{x}\right] } & =\left[T_{y}, R_{y}\right]=\left[T_{z}, R_{z}\right]=0,
\end{aligned}
$$

and therefore

$$
\mathfrak{g}=\operatorname{Vect}\left\{T_{x}, T_{y}, T_{z}, R_{x}, R_{y}, R_{z}\right\}
$$

is a 6-dimensional Lie algebra. Let us consider the system

$$
\dot{p}=T_{z}(p)+u T_{x}(p)+v R_{z}(p),
$$

where $p \in S^{2}$ and $u, v \in \mathbb{R}$.

Its Lie algebra $\mathcal{L}$ and its zero-time ideal $\mathcal{L}_{0}$ are both equal to $\mathfrak{g}$, and the rank condition is satisfied. The system is therefore diffeomorphic to a linear system on a homogeneous space. Yet it is not controllable. Indeed the equator $\{z=0\}$ is invariant for $T_{x}$ and $R_{z}$, and on this set the field $T_{z}$ points toward the north. This shows that the reachable set of a point $p_{0}=(x, y, 0)$, with $x^{2}+y^{2}=1$, belonging to the equator is contained in the northern hemisphere. More accurately it is easy to see that the reachable sets are

$\mathcal{A}(p)=S^{2}$ if $p$ belongs to the southern hemisphere;

$\mathcal{A}(p)=\{p\} \cup\{$ the northern hemisphere $\}$ if $p$ belongs to the equator;

$\mathcal{A}(p)=\{$ the northern hemisphere $\}$ if $p$ belongs to the northern hemisphere. 
Finally the Lie algebra $\mathcal{L}$ is isomorphic to the real Lie algebra $\mathfrak{s o}(3, \mathbb{C})$. Let us first consider the algebra $\mathfrak{s o}(3, \mathbb{R})$. It is generated by the matrices

$$
E_{1}=\left(\begin{array}{ccc}
0 & 0 & 0 \\
0 & 0 & -1 \\
0 & 1 & 0
\end{array}\right), \quad E_{2}=\left(\begin{array}{ccc}
0 & 0 & 1 \\
0 & 0 & 0 \\
-1 & 0 & 0
\end{array}\right), \quad E_{3}=\left(\begin{array}{ccc}
0 & -1 & 0 \\
1 & 0 & 0 \\
0 & 0 & 0
\end{array}\right),
$$

which satisfy (for the bracket $[A, B]=A B-B A$ ):

$$
\left[E_{1}, E_{2}\right]=E_{3}, \quad\left[E_{2}, E_{3}\right]=E_{1}, \quad\left[E_{3}, E_{1}\right]=E_{2}
$$

Consequently $\mathfrak{s o}(3, \mathbb{C})$ is a 6 -dimensional real Lie algebra, a basis of which is

$$
E_{1}, \quad E_{2}, \quad E_{3}, \quad i E_{1}, \quad i E_{2}, \quad i E_{3} .
$$

The isomorphism between $\mathcal{L}$ and $\mathfrak{s o}(3, \mathbb{C})$ is obtained by the identifications

$$
R_{x} \sim-E_{1}, \quad R_{y} \sim-E_{2}, \quad R_{z} \sim-E_{3}, \quad T_{x} \sim-i E_{1}, \quad T_{y} \sim-i E_{2}, \quad T_{z} \sim-i E_{3}
$$

As in the previous counterexample the algebra $\mathcal{L}$ is semisimple non compact, and the vector field $T_{z}$ is not Poisson stable.

\section{Appendix. Density on a MANifold}

A good general reference here is [2].

Let $E$ be a $n$-dimensional real vector space. A density on $E$ is defined as the absolute value $\delta=|\alpha|$ of a non-vanishing $n$-linear form $\alpha$.

Let us denote by Dens $(E)$ the set of densities of $E$. If $\delta_{0}$ is one of them, then $\operatorname{Dens}(E)=\mathbb{R}_{+}^{*} \delta_{0}$. The canonical density of $\mathbb{R}^{n}$ is denoted by $\delta_{n}=\left|\mathrm{d} x_{1} \wedge \cdots \wedge \mathrm{d} x_{n}\right|$.

An isomorphism $f$ from $F$ onto $E$ determines a density on $F$ by $f^{*} \delta=\left|f^{*} \alpha\right|$ (with $\left.\delta=|\alpha|\right)$. In particular whenever $E=F$ we have

$$
f^{*} \delta=|\operatorname{det}(f)| \delta
$$

Let $M$ be a manifold. The fiber bundle $\cup_{x \in M} \operatorname{Dens}\left(T_{x} M\right)$ is denoted by $\operatorname{Dens}(M)$, and a density on $M$ is a section of Dens $(M)$ : it is locally the absolute value of a form volume.

A continuous density $\delta$ on $M$ determines canonically a positive Radon measure, also denoted by $\delta$, in the following way: let $(U, \varphi)$ be a local chart of $M$ and let $f$ be a compactly supported continuous function. If the compact support of $f$ is included in $U$, then

$$
\delta(f)=\int_{M} f \delta=\int_{\varphi(U)}\left(f \circ \varphi^{-1}\right)(x) a(x) \mathrm{d} x_{1} \ldots \mathrm{d} x_{n},
$$

where $\left(\varphi^{-1}\right)^{*} \delta=a \delta_{n}$. The extension to $\mathcal{C}_{c}(M)$ is standard.

Let $\Phi$ be a diffeomorphism from the manifold $N$ onto $M$. Then for every compactly supported continuous function $f$ on $M$ the following equality holds:

$$
\int_{N}(f \circ \Phi) \Phi^{*} \delta=\int_{M} f \delta
$$




\section{REFERENCES}

[1] H. Abbaspour and M. Moskowitz, Basic Lie Theory. World Scientific (1997).

[2] M. Berger and B. Gostiaux, Géométrie différentielle: variétés, courbes et surfaces. Presses universitaires de France (1987).

[3] A. Borel, Compact Clifford-Klein forms of symmetric spaces. Topology 2 (1963) 111-122.

[4] F. Cardetti and D. Mittenhuber, Local controllability for linear control systems on Lie groups. J. Dyn. Control Syst. 11 (2005) 353-373.

[5] J.P. Gauthier, Structure des systèmes non linéaires. Éditions du CNRS, Paris (1984).

[6] S. Helgason, Differential Geometry and Symmetric Spaces. Academic Press (1962).

[7] Ph. Jouan, Equivalence of control systems with linear systems on Lie groups and homogeneous spaces. ESAIM: COCV 16 (2010) 956-973.

[8] Ph. Jouan, Finite time and exact time controllability on compact manifolds. J. Math. Sci. (to appear).

[9] V. Jurdjevic, Geometric control theory. Cambridge University Press (1997).

[10] V. Jurdjevic and H.J. Sussmann, Control systems on Lie groups. J. Differ. Equ. 12 (1972) 313-329.

[11] C. Lobry, Controllability of nonlinear systems on compact manifolds. SIAM J. Control. 12 (1974) 1-4.

[12] G.D. Mostow, Homogeneous spaces with finite invariant measure. Ann. Math. 75 (1962) 17-37.

[13] V.V. Nemytskii and V.V. Stepanov, Qualitative Theory of Differential Equations. Princeton Uniersity Press (1960).

[14] Yu.L. Sachkov, Controllability of invariant systems on Lie groups and homogeneous spaces. J. Math. Sci. 100 (2000) $2355-2427$.

[15] L. San Martin and V. Ayala, Controllability properties of a class of control systems on Lie groups, Nonlinear control in the year 2000 1, Paris, Lecture Notes in Control and Inform. Sci. 258. Springer (2001) 83-92. 